\title{
\begin{tabular}{l|l} 
POLITIQUES \& & Politiques et management public
\end{tabular}
}

\section{La ruelle, le tuyau et le jardinet : le partage public - privé comme objet d'un travail}

The street, the pipe and the garden: the boundary between public and private sector as a research object

Gilles Jeannot

\section{(2) OpenEdition}

\section{Journals}

Édition électronique

URL : http://journals.openedition.org/pmp/1255

DOI : 10.4000/pmp.1255

ISSN : 2119-4831

Éditeur

Institut de Management Public (IDPM)

Édition imprimée

Date de publication : 1 mai 2008

Pagination : 31-42

ISSN : 0758-1726

Référence électronique

Gilles Jeannot, «La ruelle, le tuyau et le jardinet : le partage public - privé comme objet d'un travail », Politiques et management public [En ligne], Vol. 26/1 | 2008, mis en ligne le 26 avril 2011, consulté le 19 avril 2019. URL : http://journals.openedition.org/pmp/1255 ; DOI : 10.4000/pmp.1255

(c) Tous droits réservés 


\section{LA RUELLE, LE TUYAU ET LE JARDINET : \\ LE PARTAGE PUBLIC - PRIVÉ COMME OBJET D'UN TRAVAIL}

\section{Gilles JEANNOT *}

Résumé Comment la quête d'une réponse efficace à une prescription conduit-elle à une activité créative de redéfinition des frontières entre le public et le privé ? L'article présente les discussions autour de trois projets successifs d'aménagement d'une rue d'un quartier résidentiel urbain. L'opposition entre le premier projet d'alignement pour faciliter le transit routier et un second projet dit de "cour urbaine " permet de mettre en évidence l'évolution au cours du temps de la conception de ce qui fait de cette ruelle un objet public. L'opposition entre le second projet et la solution finalement retenue permettra de répondre à la question initiale. En effet, au sein d'une conception partagée de ce bien commun, une solution à un conflit ancien ne sera trouvée qu'en bousculant les règles de la domanialité publique, et de manière toute pratique en faisant passer une canalisation d'assainissement sous des terrains privés.

* Université Paris Est, École nationale des ponts et chaussées, LATTS

Revue POLITIQUES ET MANAGEMENT PUBLIC, Volume 26, n 1. 2008.

(C) Institut de Management Public - 2008. 
Introduction La quête d'une réponse efficace à une prescription peut parfois passer par une plus grande autonomie laissée aux agents qui, au plus près du «terrain », sont les mieux à même d'ajuster l'intervention aux conditions locales. C'est ainsi que dans des secteurs divers de l'action publique, projets expérimentaux, fonctions ouvertes de médiation, qualification juridique, le travail ${ }^{1}$ des agents, leur capacité à résoudre en situation des problèmes ou à stabiliser un accord, s'est imposé comme une composante de la réussite (Jeannot, 2008). Dans cette réinvention locale, l'atteinte des objectifs peut nécessiter, plus que de simples " ajustements", des recompositions substantielles de caractéristiques fondamentales de l'intervention et en particulier des extensions du domaine légitime de l'intervention publique. Ainsi la quête d'efficacité peut-elle conduire à une activité créatrice de redéfinition des frontières entre le public et le privé.

Derrière le thème de la frontière entre public et privé on peut lire deux questions. La première, qui a occupé le débat sur le devenir des services publics porte sur le choix de l'opérateur pour la prise en charge d'une finalité publique (prestation en régie, prestation déléguée ou privée régulée). La seconde, mobilisée ici, concerne en amont de cette question le domaine d'extension de l'intervention de l'acteur public ${ }^{2}$. Cette frontière là, souvent à peine aperçue, peut traverser notre vie quotidienne. Le choix des dessins des faïences de notre salle de bains est une affaire privée, celui de la couleur des volets ou de la couverture de notre maison peut faire l'objet de réglementations publiques. Et pour être aussi familières, elles trouvent souvent leur origine dans la longue durée, c'est ainsi que pour cet exemple on pourrait remonter plus d'un siècle en amont et se souvenir que Victor Hugo ouvrait une brèche dans le caractère privé de la propriété immobilière en déclarant que si une maison appartient à son propriétaire, sa beauté appartient à tous.

La délimitation du tracé de la frontière, entendue au second sens, peut se jouer dans des débats philosophiques (par exemple la discussion sur les principes régissant l'usage thérapeutique des cellules souches), être l'objet de théories économiques comme celle des biens publics, elle peut aussi bien sûr être discutée dans l'espace de la confrontation politique et se traduire par des règles nouvelles de droit (l'entrée de la tenue des collégiennes dans la sphère publique après l'affaire du "voile islamique », par exemple). Elle peut enfin se redéfinir dans le travail quotidien des agents publics et dans la quête de solutions pragmatiques, ce qui nous préoccupe ici.

Ces différentes modalités de glissements de la frontière sont souvent entremêlées. Le cas retenu, une discussion sur l'aménagement d'une ruelle en périphérie urbaine, bien que fort modeste, a la vertu de permettre d'isoler la

\footnotetext{
${ }^{1}$ Le terme travail a pris dans le sens commun une très large extension allant de l'activité à l'emploi et même aux valeurs de la société qui s'est construite autour de l'activité productive. Nous nous attacherons cependant ici à un usage plus limité de ce terme en portant l'accent sur deux caractéristiques. En premier lieu le fait que tout travail suppose de capacité locale de l'individu à résoudre en situation des problèmes, le travail entendu au sens de Dejours (1994), comme confrontation à ce qui résiste, de Zarifian (1995) comme traitement de l'événement ou de Moisdon (1994) comme ce qui reste après que l'on ait appliqué les prescriptions fixées par les gestionnaires. En second lieu, le caractère hétéronome du travail et le fait qu'il lui est assigné une visée d'efficacité (Clot, 1999).

${ }^{2}$ La notion de publicness semble avoir été principalement été mobilisée en théorie des organisations pour évoquer la première (Bozeman, Bretschneider, 1994), mais elle est employée dans le sens commun pour évoquer le deuxième sens (Dewey, 1927).
} 
manière dont la frontière se redéfinit dans un travail, d'autres raisons comme l'évolution sociétale des représentations de la frontière légitime. En stylisant une histoire plus complexe, nous pouvons dégager trois solutions techniques d'aménagement de cette ruelle. L'opposition entre la première et la deuxième permet de mettre en évidence la manière dont la représentation de ce bien public a évolué de manière globale dans la société en quelques décennies et comment cela s'est traduit par des partis d'aménagement différents. L'opposition entre la seconde et la troisième permet -au sein d'une même conception de ce bien public- de pointer comment la sortie du conflit passe par le travail fin de recomposition des frontières accompli par un chargé de mission de la ville.

Le

réaménage-
ment de la
rue du
docteur
François à
Strasbourg

La rue du Docteur François à Strasbourg ${ }^{3}$ s'inscrit dans une ancienne zone d'extension de crues qui servait de jardin maraîcher pour la ville. Cette rue est bordée de petites maisons de villes sur deux niveaux, précédées de jardinets arborés et clôturés. La rue est étroite, guère plus de $3 \mathrm{~m}$ à certains endroits, et sinueuse. Ici, des renfoncements où la voie est particulièrement resserrée, là des passages plus larges permettant quelques stationnements.

Les habitants sont très attachés au charme de cette rue qui s'inscrit dans l'exvillage de la Robertseau, et une association très vivante regroupant l'essentiel des habitants existe depuis des années. Cette association maintient le lien entre les habitants en organisant régulièrement des animations. Un conflit oppose depuis plusieurs décennies cette association et les services de la ville. Ceci a conduit à laisser sans entretien cette ruelle.

La controverse sur cet aménagement est ancienne. Pour ce qui concerne la problématisation des interdépendances ${ }^{4}$ dans lesquelles cette rue est engagée et les solutions proposées, trois périodes se dégagent. La première, dans un contexte où les débats sont dominés par la question du trafic de transit, est le moment d'un projet d'alignement assez contraignant. La seconde voit apparaître une plus grande valorisation de la dimension patrimoniale de cette rue et un autre facteur d'interdépendance lié celle fois non plus à la circulation des voitures mais à l'évacuation des eaux usées. Une option technique nouvelle dite de la « cour urbaine » qui permet de réduire l'espace approprié par le public est alors soumise à la discussion. Durant la troisième enfin, dans le même cadre de problématisation des interdépendances, une forme de bricolage conduit à faire éclater les principes traditionnels de partage de la domanialité.

\footnotetext{
3 Je remercie Claude Narioo d'avoir attiré mon attention sur ce cas.

4 John Dewey (1927), dans son ouvrage The Public and it's problems nous offre un cadre général pour aborder la justification du partage public privé. II illustre son propos par un fait très élémentaire la discussion entre deux entrepreneurs. A priori celle-ci est une affaire privée qui ne concerne pas les pouvoirs publics, mais qu'il s'agissent d'entrepreneurs du même domaine, en position dominante et celle-ci pourrait soudain préfigurer une "entente" et cette discussion devient de nature publique. Derrière cet exemple il met en avant le critère de l'interdépendance qui fonde selon lui la ligne de partage.
} 


\section{Première période : le projet d'élargissement lié au transit}

La première phase qui débute dès les années 1970, oppose deux lectures de l'enjeu du caractère public de la rue. D'un côté les services de voirie de la ville de Strasbourg cherchent à multiplier les voies de transit vers le nord est de l'agglomération et de l'autre les habitants demeurent attachés à un usage public limité aux riverains de la rue. La question de l'interdépendance est donc posée par les services de la voirie qui ont en vue la circulation générale de la ville et considèrent cette rue comme un espace appropriable par les automobilistes des quartiers du nord est. Ils peuvent alors dénoncer l'appropriation égoïste par les riverains de cet espace public, potentiellement utile à l'ensemble des habitants de la ville, en opposant à la qualité de vie de quelques uns, la perte de temps de beaucoup d'autres.

La discussion sur le projet proposé par la ville se focalise alors sur une question de profil en travers, c'est-à-dire la vue en coupe type de la voirie projetée. Le plan d'occupation des sols prévoit ainsi un alignement pour une voirie de $10 \mathrm{~m}$ de large, ce qui conduit de fait à supprimer les jardinets devant les maisons. Mais les habitants s'y sont toujours opposés et une association très active a régulièrement contré ces projets du service voirie de la ville de Strasbourg.

Une telle situation qui oppose un intérêt général de la circulation à la protection des habitants de la ruelle aurait pu conduire à un «forçage» (Mermet et alii, 2004) de l'administration par le canal d'expropriations générales. Cependant du fait de l'état du rapport entre l'enjeu (relativement limité tout de même) et la détermination des habitants, une telle méthode n'a pas été retenue. Les services de la mairie ont pris le parti d'une intervention progressive. Au fil du temps, des acquisitions de terrains privés ont été réalisées pour permettre à terme un alignement de la voirie. Deux tiers des jardins de devant des petites propriétés ont ainsi été acquis. Ce processus est cependant assez lent. Les services de la ville vont alors être rattrapés par un regard nouveau porté sur l'environnement.

\section{Deuxième période : le projet de « cour urbaine »}

La manière d'aborder le cas de la rue du docteur François a été marquée par deux faits nouveaux. D'une part, une évolution notable du statut de la circulation automobile urbaine qui atténue la tension entre fonction de transit et fonction résidentielle au cœur du conflit antérieur. D'autre part l'ajout d'une contrainte supplémentaire imposée par la loi sur l'eau qui rend visible un autre facteur d'interdépendance et qui conduira à une nouvelle configuration d'acteurs plus favorable au déblocage de la situation.

Le conflit à propos de la fonction de transit de la voie s'est progressivement apaisé. Entre temps, en effet, la question de la voirie s'est posée en des termes différents. Avec l'émergence des préoccupations environnementales, et en particulier de la loi sur l'air et des plans de déplacement urbains, le parti pris de favoriser la circulation automobile par tous les canaux possibles est tombé progressivement en défaveur. Alors que permettre la circulation par tous les canaux était une valeur en soi, la rendre plus difficile devient un objectif au moins implicite de bien des politiques d'aménagement urbain. C'est ainsi que, 
dans le cadre des plans de déplacements urbains (Offner, 2006), la réduction des places de stationnement, l'occupation de la voie publique par des transports en commun en site propre sont apparues aux municipalités comme les moyens les moins controversés de réduire l'usage de l'automobile et de peser sur le transfert modal vers les transports en commun. En outre, des préoccupations croissantes concernant la sécurité des piétons et tout particulièrement des enfants se sont ajoutées à la mise en cause de l'usage de la trame viaire de desserte résidentielle pour le transit et ont conduit à différentes modalités de "pacification" de la circulation. Par ailleurs, la nouvelle politique de transports urbain valorise les possibles usages ludiques d'une telle voirie pour des promenades, en particulier en vélo. Ceci relie la rue aux habitants d'autres quartiers mais en des termes moins conflictuels. II y a en effet dans cette nouvelle approche plus aisément composition des usages entre l'accès en voiture pour les riverains et la circulation de bicyclettes pour la promenade. Au final, ce qui était considéré comme une appropriation indue de l'espace public destiné au transit peut devenir plus aisément appréhendé comme une utile co-production d'une nouvelle manière de vivre les déplacements 5 .

La loi sur l'eau et les obligations de résultat en matière de collecte des eaux usées et de pollution des cours d'eaux met par ailleurs à jour un problème ancien de cette rue. Celle-ci, n'a jamais été reliée au réseau d'égout. Chaque maison dispose d'un assainissement autonome. Certaines rejettent leurs eaux sales dans un puits perdu, d'autres disposent d'une fosse vidée à intervalles réguliers. Ceci crée des désordres publics pour les riverains (odeurs, blocage de la voie pour les vidanges) et surtout cela peut avoir des conséquences importantes pour le reste de la population de la ville qui partage la même nappe phréatique. Les maisons sont reliées par le sous-sol à un autre bien commun. Ceci repose la question du partage entre le privé et le public à travers non plus la figure horizontale de l'espace de transit, mais la figure verticale du risque de contamination. Cette question s'impose progressivement avec la prise de conscience de l'importance des enjeux d'environnement et de santé publique. Elle se cristallise plus directement autour des normes nouvelles imposées par la loi sur l'eau qui imposent aux habitants, dans un milieu aussi dense, le raccordement à un réseau collectif ${ }^{6}$.

Ce second argument (sur l'eau) portée dans la mairie par le service assainissement va être l'occasion pour les responsables du service de la voirie de revenir sur le sujet douloureux, pour eux, de cette ruelle. En effet, les objectifs de réaménagement liés à l'installation d'un collecteur des eaux usées sont convergents avec ceux du service de voirie dans la mesure où le passage d'un tuyau de collecte suppose une reprise globale de la voirie et des réseaux associés. L'inscription du tuyau de collecte des égouts sur la voie existante est impossible du point de vue des normes techniques, plusieurs réseaux sont déjà

\footnotetext{
${ }^{5}$ Cette évolution générale de la doctrine sur la circulation automobile en ville ne fait pas l'unanimité et certains voient derrière les arguments d'environnement et de sécurité un prétexte pour masquer l'appropriation par les habitants d'un espace possible de circulation. La question a en particulier été posée dans le cas parisien autour du droit des banlieusard de circuler dans Paris intra-muros.

${ }^{6}$ En 1992, la loi sur l'eau avait instauré l'obligation pour la ville de réaliser avant 2000 un plan de zonage qui précise la répartition géographique des zones d'assainissement collectif et de celles d'assainissement individuel.
} 
présents sous la chaussée (électricité, eau) et chacun suppose une largeur minimale de protection.

C'est alors une autre solution que propose le service de la voirie pour intégrer à la fois les nouvelles contraintes liées à la loi sur l'eau et la nouvelle approche de l'usage de la circulation dans les espace résidentiels: la "cour urbaine». Le terme " cour urbaine » est une transcription du néerlandais "woonerf » (cour d'habitants) et correspond à des expériences fondatrices dans ce pays. II s'inscrit dans un ensemble plus ou moins convergent d'expériences visant à créer un espace de circulation partagé entre les différents usagers et en particulier les voitures et les enfants, home zone (rues résidentielles), childstreet (rue pour les enfants), verkehrsberuhigtestrasse (rue à circulation apaisée). Concrètement la cour urbaine se présente comme un espace indifférencié ouvert aux piétons et aux voitures. Objet de discussions dans les milieux spécialisés depuis des années, cette solution technique a progressivement été codifiée par les autorités compétentes. La « cour urbaine » se caractérise par un profil en travers en $\mathrm{V}$ avec un caniveau au milieu, des encoches pour les stationnements, le tout couvert de pavés qui marquent le statut mixte (piétons/voitures) de la voirie.

L'emploi de l'expression « cour urbaine » évoque bien une certaine ambiguïté entre la sphère du privé (la cour) et celle du public (urbaine). Cette ambiguïté trouve son origine dans la tradition néerlandaise d'un statut domanial mixte de l'espace au devant des maisons. " $A u 17^{\mathrm{e}}$ une sorte de trottoir, appelé " stoep » était aménagé juste devant les maisons ; il était légèrement surélevé par rapport à la « chaussée » et permettait à la fois le jeu des enfants, l'étalage des marchandises, la conversation et le repos sur les bancs fréquemment accolés aux façades » (Loiseau, 2005). Cet espace prolongé par des escaliers menant aux maisons, approprié de manière semi-privative, sera revendiqué par la suite par les tenants de la cour urbaine. Ceci dit, pour la solution technique proposée, l'ambiguïté n'est que symbolique : l'espace partagé entre voitures et piétons est clairement de domanialité publique. L'innovation principale de la cour urbaine est de faire coexister au sein de l'espace public les droits et devoirs des piétons et des voitures, ce qui se traduit par un nouveau « code de la route $»$.

Pour revenir au cas de la rue du docteur François, cette solution technique offre un modèle standardisé qui suppose une uniformisation de la largeur de la voirie à $5 \mathrm{~m}$. Les habitants refusent une telle solution depuis le début. Ils vont continuer à s'opposer au projet.

\section{Troisième période : les tuyaux sous les jardinets}

La solution finale qui lèvera le blocage ne peut être comprise sans l'intervention d'un nouvel acteur. La municipalité de Strasbourg a engagé en 1995 une politique territoriale de proximité. Le parti pris, que l'on retrouve à la même époque dans différentes villes (Montreuil ; Nantes, ...) est de mieux relier les pratiques de démocratie participative et l'offre des services techniques. Les élus, dont le président du comité de quartier adjoint au maire, décident en 1999 d'engager une démarche innovante de dialogue avec les riverains et les associations du quartier pour l'aménagement de la rue. L'organisation de ce 
dialogue est prise en charge par le nouveau service de l'action territoriale. Celle-ci regroupe des chargés de mission par quartier qui ont pour tâche de faciliter le fonctionnement des lieux de débat public en maintenant un lien actif avec les associations ou comités de quartier et en aidant les élus à affronter des rencontres parfois conflictuelles avec la population. Mais ils ont aussi pour objectif de permettre des réponses plus rapides en facilitant le lien avec les services techniques réorganisés partiellement sur le même découpage territorial que celui des instances de participation. II est remarquable de noter que cette organisation généralise des expériences menées dans les quartiers populaires de HLM à toute la ville et qu'une bonne part des chargés de mission, ont un profil de chargé de développement social urbain. C'est le cas du chargé de mission attaché à ce quartier qui s'est vu affecter le travail de médiation sur ce dossier durablement enlisé.

Ce chargé de mission va intervenir dans un contexte plus favorable à l'obtention d'un accord. D'une part la nouvelle municipalité s'est montrée plus sensible aux demandes de l'association, d'autre part, surtout, le service assainissement a des contraintes plus forte que le service voirie. En effet tant les directives européennes que la loi sur l'eau lui imposent des contraintes de résultat avec des échéances qui se rapprochent progressivement. Cela le rend plus enclin à transiger sur les normes techniques pour aboutir.

Cependant ces conditions favorables ne suffisent pas à faire apparaître la solution qui sera retenue dans son principe et dans ses modalités précises. Son intervention sera l'objet d'un complexe travail d'animation d'une réflexion collective et de négociation entre les habitants et les services techniques pour caler le projet. La contribution de ce chargé de mission va être de permettre la convergence des différentes parties sur une nouvelle manière de poser le problème, et ceci en déplaçant un a priori jusque là non discuté sur la frontière entre l'espace public et l'espace privé. Cela va aussi être de rendre opérationnelle cette nouvelle orientation en rediscutant maison par maison les conditions du nouveau tracé de la frontière.

Pratiquement, la nouvelle solution conduit à faire passer la canalisation de collecte des égouts dans les jardinets qui séparent les maisons de la voirie et qui sont de propriété privée. La solution est globalement moins coûteuse (on évite de refaire complètement la voirie défoncée par une tranchée), mais elle suppose un travail d'ajustement plus fin pour faire accepter la nouvelle solution technique aux habitants et l'adapter aux singularités de chaque propriété. Comment la tranchée sera-t-elle recouverte (sablé ou terre végétale), comment les clôtures seront-elles rétablies (clôture arbustive ou croisillon métal) ? (ils choisiront la terre végétale et la haie de feuillus) que faire pour les arbres qu'il faudra abattre pour permettre le passage du tuyau ?

$\grave{A}$ travers ces discussions se dessine une ligne de partage fine entre ce qui relève de l'espace public et de l'espace privé. Tout d'abord, bien sûr, le fait de faire transiter une infrastructure publique sur un terrain privé. C'est là aller contre la division implicite des espaces et des fonctions. Mais il n'y a pas que cela. La discussion se fera sur l'idée d'une valeur publique de l'atmosphère liée à la qualité des jardins privés. Les habitants s'efforcent ainsi de caractériser l'aspect un peu " brouillon » et irrégulier de cette rue. Dans certains cas, des arbres, supprimés sur une propriété et correspondant à une perte pour 
I'habitant qui en a directement jouissance, seront remplacés par des plantations nouvelles sur le terrain public à proximité. Dans d'autres cas, comme pour marquer l'entrée de la rue et signifier que la vitesse maximale n'est plus à cinquante kilomètres heure mais à trente, les services municipaux planteront sur des terrains privés des arbres qui symboliseront le statut de la voie. Cela inclut aussi de prendre en compte la possibilité à long terme d'interventions pour les services de l'assainissement sur les terrains privés et celle de contrôler en partie l'usage de ce terrain (par exemple empêcher la plantation d'arbres dont les racines détérioreraient le tuyau d'assainissement). Tout le travail consiste ici à faire se recouvrir les territoires du public et du privé. L'acteur public s'étend sur le domaine privé. La qualité paysagère est construite comme une coproduction. Le chargé de mission de la ville établit une liste des arbres privés comme une liste de patrimoine commun. Et il reconnaît un droit de regard des habitants et une certaine appropriation par eux de l'espace public. Après cet accord et les travaux réalisés, la ville revendra les jardinets qu'elle avait acquis au cours du temps, mais seulement pour la partie non concernée par la canalisation ${ }^{7}$. Ceci fait que, dans ces cas là, un espace de propriété publique se trouve derrière la clôture et apparaît au passant comme un espace privé, alors que, dans les cas où la ville n'avait pas racheté précédemment, un terrain privé supporte une servitude publique. Curieusement ce caractère entremêlé des statuts de propriété rejoint une spécificité historique de cette rue liée au croisement des droits germanique et français ${ }^{8}$.

Ici, les caractéristiques de la rue résistent, conjointement avec les habitants, aux solutions standardisées proposées depuis des années par les ingénieurs du service de la voirie. Passer en force serait détruire un bien public propre à la rue, son atmosphère originale. En essayant de le préserver, notre interlocuteur découvre que ce bien public est constitué en partie d'éléments privés et en partie d'éléments publics. Agir suppose de trouver une nouvelle frontière entre public et privé. Cela ne se joue pas hors de toute contrainte. II y a tout d'abord la loi sur l'eau qui oblige à sortir du statu quo. Les questions financières ne sont pas négligeables. II y a aussi tout un ensemble de dispositions pratiques liées au rétablissement des clôtures existantes ou au fait que les canalisations ne cohabitent pas facilement avec la végétation. Le travail consiste alors à imaginer une solution qui puisse intégrer ces différentes contraintes, à intéresser les différentes parties (les habitants, le service assainissement) mais aussi, à partir d'une solution assez solide, à obtenir l'arbitrage des élus en défaveur du service de la voirie. Ce faisant, lorsque le service d'assainissement aura posé sa canalisation, que des arbres auront été coupés et d'autres replantés, qu'un document fixant les engagements de chacun aura été rédigé,

\footnotetext{
7 On peut noter qu'ainsi, les habitants concernés ne s'approprient pas la plus value générée par l'aménagement puisqu'ils ont vendu aux conditions antérieures à l'aménagement pour racheter au tarif postérieur à l'aménagement.

${ }^{8}$ "Dans la rue du Docteur François en l'occurrence je me souviens d'un couple qui propriétaire de leur terrain m'avait contacté pour s'inquiéter des dégâts produits par l'intervention des services de la communauté urbaine de Strasbourg sur leur propriété. Après m'avoir invité pour constater la largeur de la tranchée et la destruction de leur cerisier "japonais" la dame m'avait ouvert ses papiers notariés contenant l'acte de propriété. Celui-ci était pour partie rédigé en allemand gothique. Transmis au service des affaires immobilières il n'y eu plus aucun motif de recours pour les propriétaires. La raison était je crois que dans l'acte de propriété figurait une close "d'utilité commune" d'une partie de la parcelle à fin de "service commun". » (interview, chargé de mission).
} 
ce délicat mélange entre espace public et privé, qui fait l'atmosphère de cette ruelle, aura été préservé au prix d'un nouveau tracé de la frontière entre ces deux composantes.

La

redéfinition de la frontière comme travail
La mise en regard des deux premières figures de projet permet de souligner l'évolution dans le temps des conceptions de ce qui est public. Dans les premiers projets de réalignement de la rue, le fait public central était la possibilité d'une circulation routière dans la voie. II s'agissait de préserver la possibilité pour des voitures de non riverains de traverser ce quartier ou pour les services publics (comme la collecte des ordures) de s'accomplir sans encombrement. La question de l'évacuation des eaux usées n'est alors pas absente, une réfection de la ruelle aurait été l'occasion de rénover l'ensemble des «voiries et réseaux divers», l'eau, les égouts, l'électricité, le téléphone. Mais elle est alors secondaire. Dans la seconde période l'interdépendance saillante n'est plus le potentiel de transit routier de la rue mais le fait que les pertes des puits perdus risquent de polluer la nappe phréatique. En outre, pour ce qui concerne l'espace public de surface, c'est une fonction d'agrément, pour les riverains, mais aussi pour les promeneurs extérieurs qui s'impose. La proposition de "cour urbaine» atténue alors le caractère fonctionnaliste des premiers projets en permettant la cohabitation dans le même lieu des enfants qui jouent, des cyclistes et des voitures dont la vitesse aura été strictement réduite.

Cependant, si on se place du côté de la méthode d'intervention et de la manière de tracer la frontière entre le public et le privé, ces deux projets apparaissent très proches. En effet dans les deux cas, et malgré l'évolution notable des critères de définition de ce qui relève du public, la démarche est la même. Les critères conduisent à définir un cahier des charges des prestations à accomplir, circulation des voitures individuelles et des services publics (ex pompiers), stationnement, circulation des piétons, installation en sous sol ou en aérien des réseaux. La somme de ces contraintes gérées au mieux dans le respect de normes techniques établies dans la longue durée (par exemple les réseaux ne peuvent être superposés, les pompiers imposent une largeur minimale et un accès à chaque habitation), conduit à un dimensionnement minimal de l'espace public. Et dans le cas où cet espace minimum dépasse le domaine public existant, il s'agit de rogner sur telle ou telle propriété privée.

L'opposition de l'exemple de la cour urbaine et de la solution finalement retenue permet alors de mettre en évidence ce qui a changé. En effet du point de vue des principes de ce qui relève du public et du privé, il y a bien consensus : la questions de la pollution de la nappe est reconnue comme un problème important et la ruelle ne se voit plus attribuer de fonction de transit. Du point de vue du paysage urbain finalement obtenu, le résultat n'aurait pas été très différent non plus mis à part l'effet d'alignement à certains endroit et quelques «tics» d'aménageurs (pavés autobloquants, profil en $\mathrm{V}$, lampadaire néo-vieillots, etc.). Le jogger du dimanche qui passerait par là n'aurait pas vraiment vu la différence. C'est du point de vue de la méthode de partage entre public et privé que les choses ont changé. Alors que le modèle de la «cour urbaine» propose une définition normalisée de ce que doit être le partage entre public et privé, la solution proposée a conduit d'abord à mettre en discussion puis fixer les critères de ce partage (règles sur l'équivalence générale dans les arbres 
plantés, modalités de reprise des clôtures...) et enfin à négocier parcelle par parcelle avec chaque propriétaire les modalités particulières de transcription de ces principes.

Le fait que cette solution conduise à lever le blocage et que finalement le tuyau d'égout ait pu passer dans les jardinets n'est pas négligeable en la matière. L'enjeu du travail est non seulement l'ajustement local mais l'atteinte d'un résultat dans une conception de l'action publique (en l'occurrence inscrite dans la loi sur l'eau et dans les nouvelles normes européennes concernant l'épuration) qui ne se contente plus d'obligation de moyens mais réclame des effets tangibles. À l'époque des premiers projets d'alignement de la voirie l'absence de résultat était un fait assez négligeable pour que les responsables administratifs acceptent qu'il ne se passe rien pendant de longues années. Aujourd'hui, les nouvelles règles européennes imposent un deadline précis pour le raccordement au réseau. Or cette réussite n'aurait pu être atteinte sans cette activité fine de redéfinition de la frontière entre le public et le privé. Si cette redéfinition locale de la frontière entre le public et le privé est un point de passage quasi-obligé (si on laisse de côté le pur forçage) alors ce travail d'ajustement occupe une place tout à fait essentielle dans la recomposition en profondeur de l'action publique autour d'un engagement de résultat.

Le caractère anodin de l'objet n'est pas sans intérêt non plus du point de vue analytique. En effet, ces prises de position d'agents publics sur la ligne de partage entre public et privé peuvent être analysées comme l'expression de valeurs propres à l'individu ou au collectif professionnel ou peuvent être rapportées à des considérations pragmatiques concernant l'efficacité de l'action publique. On a pu ainsi montrer que certains groupes de fonctionnaires prennent parti pour une conception de l'action publique, en fonction de leurs valeurs partagées ou de leur conception de leur rôle. Le cas des fonctions de sécurité permet tout à fait d'illustrer ce point de vue (De Maillard, 2006). De même, dans le cas de l'intervention économique des collectivités locales, les options plus ou moins libérales ou interventionnistes de l'agent de développement (sur la pertinence de certains types d'aides financières par exemple) et les considérations pratiques (sur le moment opportun d'une intervention) sont difficiles à démêler. Dans de nombreuses situations ces dimensions sont co-présentes et difficilement séparables. Dans le cas abordé ici, il semble difficile de rapporter le fait de faire passer le tuyau sous les jardinets à des options personnelles fortes de cet agent, ce qui permet d'isoler cette dimension de visée pragmatique dans le partage.

Le caractère limité de cet objet n'a bien sûr pas que des vertus. Tout ceci n'estil pas qu'un petit effet à la marge, certes stimulant par les questions de principe qu'il soulève mais marginal dans son impact? Dominique Lorrain (2004), en s'appuyant sur les travaux de Kostas Chatzis (2000), prend justement l'exemple des normes techniques concernant le dimensionnement des tuyaux d'assainissement pour illustrer l'idée que l'action publique d'une mairie serait portée par des " pilotes invisibles " qui s'autonomisent et imposent aux élus et a fortiori aux habitants et fonctionnaires irrespectueux, le poids d'un outil qui "structure " l'action. Le chargé de mission serait ainsi le pot de terre qui n'aurait pu bousculer que de manière bien provisoire le pot de fer de la norme technique dédiée à la voirie urbaine. Ainsi posée la question, la réponse ne peut être que positive. En effet on montrerait aisément que dans le temps où cette 
opération dérogatoire aux normes techniques et juridiques a été menée, de multiples autres opérations dont certaines de plus grande ampleur ont été accomplies en respectant les normes techniques. Mais une telle réponse ne va plus de soi si on s'intéresse à la dynamique d'évolution de ces normes. Comme le souligne Joas (1999) si les institutions tendent à se reproduire à l'identique, c'est dans les imprévus de la confrontation pratique qui forcent à la créativité, qu'elles peuvent se transformer. Même si ces mouvement peuvent être infimes, ils ne sont pas négligeables dans la perspective de très longue durée des glissements de la domanialité entre public et privé (Bourjol, 1989). Toute la question est alors de savoir dans quelle mesure un tel micro-mouvement peut venir se relier à d'autres. Dans cette perspective, il n'est pas impossible que certains aménagements tout aussi singuliers aient pu servir de matrice à la définition de la norme technique intermédiaire de la cour urbaine. On pourrait aussi rappeler que la figure de ce chargé de mission n'est pas isolée et que plusieurs municipalités (Amiens, Rennes, Nantes, Montreuil, ...) ont jugé de la même manière nécessaire de bousculer certaines rigidités de leurs services techniques en mettant directement en relation les démarches de démocratie participative et le fonctionnement quotidien des services techniques par des dispositifs comparables.

L'exemple présenté ici peut apparaître bien ténu, tant pour l'enjeu (le réaménagement d'une petite rue qui concerne tout au plus quelque centaines d'habitants) que pour «l'exploit» mis en avant, obtenir un accord entre les habitants et les services techniques de la ville en faisant passer un tuyau de collecte des eaux usées dans les jardinets au devant des maison plutôt que sous la chaussée. Le déroulement historique du cas a cependant des vertus qui permet de mettre en évidence et de dissocier deux faits importants concernant la frontière public privé à propos de biens publics.

Tout d'abord c'est une occasion de plus de mettre en évidence la complexité et la plasticité des critères de partage entre ce qui relève de la sphère publique et de la sphère privée, le fait que les interdépendances sont à la fois objectives mais aussi l'objets d'accords construit dans la durée. C'est en effet une des caractéristiques des "biens communs» de générer des articulations complexes d'usages variés et de définitions complémentaires et contradictoires.

Ensuite, et c'était la finalité de l'article, cet exemple permet de souligner la part irréductible du travail de ce chargé de mission dans le partage finalement établi. II permet aussi de mettre en évidence le fait que ce travail ajusté correspond moins à une évolution dans la définition de ce qui est public (le fait que l'on soit passé à un modèle centré sur la proximité et la préservation du patrimoine) qu'à une nouvelle relation à l'efficacité. Si l'intervention publique se construit autour d'une visée de résultat, elle doit laisser des marges d'interprétation à ceux qui peuvent apprécier localement la faisabilité d'une solution, ce qui inclut aussi de laisser un certain flottement sur une question aussi fondamentale que la ligne de démarcation entre la sphère publique et la sphère privée. Ce partage nouveau pourra demeurer une singularité atypique, mais il pourra aussi, si il est mis en relation dans des arènes professionnelles avec d'autres initiatives comparables, servir de modèle à une nouvelle norme du partage. 


\section{BIBLIOGRAPHIE}

BOZEMAN B. BRETSCHNEIDER S. (1994), «The 'publicness puzzle' in Organization Theory : A Test of Alternative Explanations of Differences between Public and Private Organizations", Journal of Public Administration Research and Theory, vol 4., $\mathrm{n}^{\circ}$ 2, avril, p. 197-223.

BOURJOL J. (1989), Les biens communaux, voyage au centre de la propriété collective, Paris, LGDJ, $452 \mathrm{p}$.

CHATZIS K. (2000), La pluie le métro et l'ingénieur, Paris, l'Harmattan.

CLOT Y. (1999), La fonction psychologique du travail, Paris, PUF, 243 p.

DEJOURS C., MOLINIER P. (1994), «Le travail comme énigme», Sociologie du travail, 1994, Hors série, p. 35-44.

DE MAILLARD J. (2006), « Les services de prévention-sécurité à l'épreuve du politique ", Politiques et Management Public, juin, vol 24, n² 2, p. 23-39.

DEWEY J. (1927), The public and it's problems, Chicago Swallow press, trad (2003), Le public et ses problèmes, Édition Farrago/ Université de Pau.

DIV (2000), Moderniser le service public des villes, territoire et proximité, Montreuil, 2425 février 2000, édition de la DIV, 227 p.

JEANNOT G. (2008), « Les fonctionnaires travaillent-ils de plus en plus ? Un double inventaire des recherches sur l'activité des agents publics », Revue française de science politique, vol. 58, $n^{\circ} 1$, p. 119-136.

LOISEAU F. (2005), Évolution des « rues à vivre » dans quelques villes européennes, CERTU, $26 \mathrm{p}$.

LORRAIN D. (2004), « Les pilotes invisibles de l'action publique. Le désarroi du politique? " in Lascoumes Pierre, Le Galès Patrick, Gouverner par les instruments, Presses de la fondation nationale des sciences politiques, p. 163197

MERMET L., DUBIEN I., EMERIT A., LAURANS Y. (2004), "Les porteurs de projet face à leurs opposants : six critères pour évaluer la concertation en aménagement", Politiques et Management Public, vol 22, $n^{\circ} 1$, mars, p. 1-22.

MOISDON J.-C. (1994), "Appareils gestionnaires et travail ou de la lacune comme opportunité», Sociologie du travail, hors série, p. 11-19.

OFFNER J.-M., Les plans de déplacements urbains, Paris, la documentation française, 2006

ZARIFIAN P., Le travail et l'événement, Paris, L'harmattan, 1995. 